\title{
EDIOBIL
}

\section{TUBERCULOSIS IN PATIENTS WITH CHRONIC KIDNEY DISEASE: ATYPICAL PRESENTATION AND DIAGNOSTIC DILEMMA}

Ravi R. Pradhan ${ }^{1}$

${ }^{1}$ Department of Internal Medicine, Institute of Medicine (IOM), Tribhuvan University Teaching Hospital (TUTH), Kathmandu, Nepal

*Correspondence to: Dr. Ravi Ranjan Pradhan, Department of Internal Medicine, Institute of Medicine (IOM), Tribhuvan University Teaching Hospital (TUTH), Kathmandu, Nepal.

Email:drravipradhan@iom.edu.np

\begin{abstract}
Tuberculosis (TB) is a serious public health problem, and more common in developing countries like Nepal. Worldwide, it is the second most frequent cause of death from infectious disease. Patient with chronic kidney disease (CKD) under maintenance dialysis are more likely to develop TB compared to general population. Given the increasing prevalence of CKD in TB endemic areas, a merging of CKD and TB epidemics could have significant public health implications, especially in low- to middle-income countries like Nepal. Because of increased frequency of extra-pulmonary tuberculosis in patient with CKD, the clinical presentation is atypical and leads to diagnostic dilemma.
\end{abstract}

\section{EDITORIAL}

Globally, more than one in three individuals is infected with tuberculosis (TB). ${ }^{1}$ Patient with chronic kidney disease (CKD) under maintenance dialysis are 6.9 to 52.5 times more likely to develop TB compared to general population, largely due to impaired cellular immunity. ${ }^{2}$ An increased risk of TB in dialysis patient was first reported by Pradhan et $\mathrm{al}^{3}$ in 1974. Prevalence of tuberculosis in patients under maintenance dialysis has been reported to be $10.5 \%$ in India. ${ }^{4}$ The disease often affects the people of reproductive age group and frequently leads to hospitalization, thus significantly increasing National Health Service Cost. Morbidity and mortality rates of TB are high in this population, rendering TB a serious public health problem. The immunosuppressive effects of uremia may partially explain these rates. In turn, impaired immune responses may lead to atypical presentation or late presentation, delayed response to therapy and increased mortality. Moreover, nutritional status, vitamin $D$ deficiency and hyperparathyroidism contribute to impaired immunity in a patient with CKD.

Clinical presentation of TB in CKD patients is often insidious and atypical. Patients frequently present with systemic symptoms, such as fever, anorexia, and weight loss. These symptoms may mimic uremia and can result in a delay of diagnosis. Extrapulmonary TB is more common than pulmonary TB; accounting for 60 to $80 \%$ cases and among extrapulmonary TB, tubercular lymphadenitis is the most common. ${ }^{5}$

Diagnosis of tuberculosis in dialysis patient can be complicated and difficult because of the increased frequency of extra-pulmonary involvement, leading to atypical manifestations and non specific symptoms.

Given that a high prevalence of TB in patient with $C K D$, this is to ensure that the treating physician or nephrologist should be aware of the unusual and varied clinical presentation TB in patient with CKD and include TB in the differential diagnosis of any patient presenting with nonspecific symptoms such as anorexia, fever and weight loss. All the efforts should be made, including invasive investigations, to reach an early diagnosis. If the results are negative and there is still a high suspicion of TB, an empirical trial of anti-tubercular therapy may be justified, especially in TB endemic areas. 


\section{REFERENCES:}

1. CDC. Tuberculosis (TB). Data and Stastistics. Available at http://www.cdc.gov/tb/stastistics/ default.htm.Accessed. June 7, 2012.

2. Andrew OT, Schoenfeld PY, Hopewell PC, Humphreys $\mathrm{MH}$. Tuberculosis in patients with end-stage renal disease. The American journal of medicine. 1980;68(1):59-65.
3. Pradhan RP, Nidus $B D$, Matalon $R$, Eisinger RP. Tuberculosis in dialyzed patients. JAMA. 1974;229:798-800.

4. Rao TM, Ram R, Swarnalatha G, et al. Tuberculosis in haemodialysis patients: A single centre experience. Indian J Nephrol. 2013;23(5):340-5

5. Romanowski K, Clark EG, Levin A, Cook VJ, Johnston JC. Tuberculosis and chronic kidney disease: an emerging global syndemic. Kidney Int. 2016;90(1):34-40. 